\title{
A carbon-based DNA framework nano-bio interface for biosensing with high sensitivity and a high signal-to-noise ratio
}

Jing Su ${ }^{a, b}, 1$, Wenhan Liu, d1 ${ }^{c}$, Shixing Chenc, Wangping Deng ${ }^{c}$, Yanzhi Dou ${ }^{c, d}$, Zhihan Zhaoc, Jianyong $\mathrm{Lic}^{c, d}$, Zhenhua Lic, d, Heng Yine, Xianting Ding ${ }^{\mathrm{b} *}$, Shiping Song a, $\mathrm{c} *$

${ }^{a}$ Shanghai Synchrotron Radiation Facility, Zhangjiang Lab, Shanghai Advanced Research Institute, Chinese Academy of Sciences, Shanghai 201210, China

${ }^{\mathrm{b}}$ State Key Laboratory of Oncogenes and Related Genes, Institute for Personalized Medicine, School of Biomedical Engineering, Shanghai Jiao Tong University Shanghai 200030, China

c Shanghai Institute of Applied Physics, Chinse Academy of Sciences, Shanghai 201800, China

${ }^{\mathrm{d}}$ University of Chinese Academy of Sciences, Beijing 100049, China

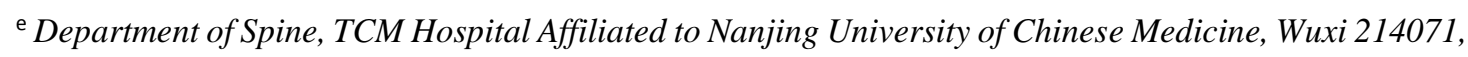
China

This file includes

Figure S1

Figure $\mathrm{S} 2$

Figure S3

Figure S4

Figure S5

Figure S6

Figure S7

Table S1

Table S2 
a)

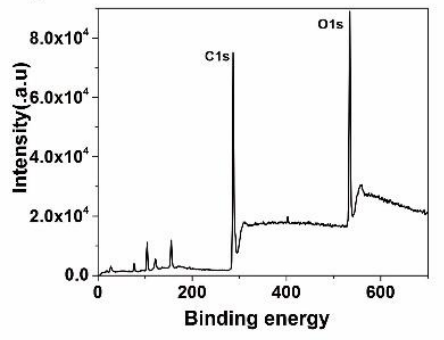

d)

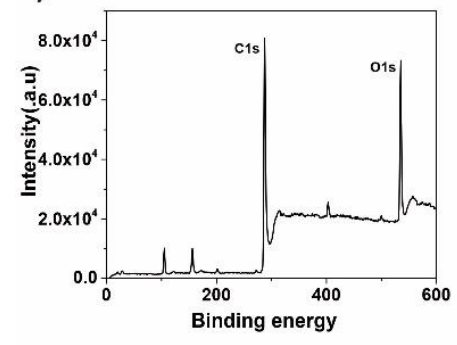

b)

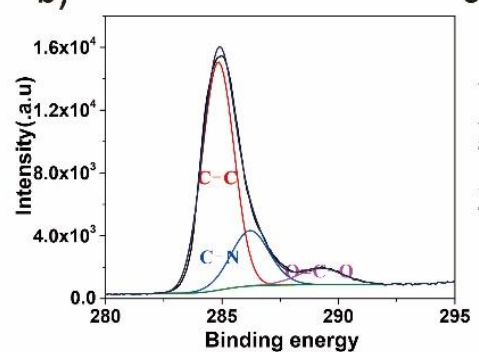

e)

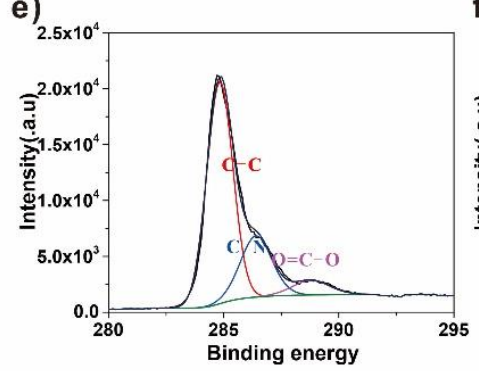

c)

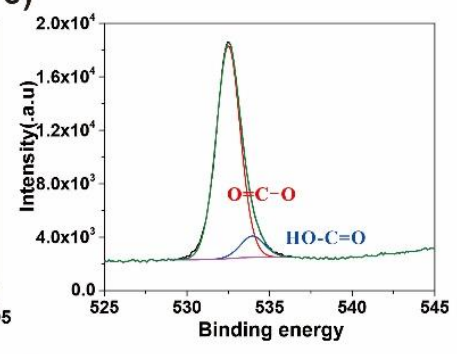

f)

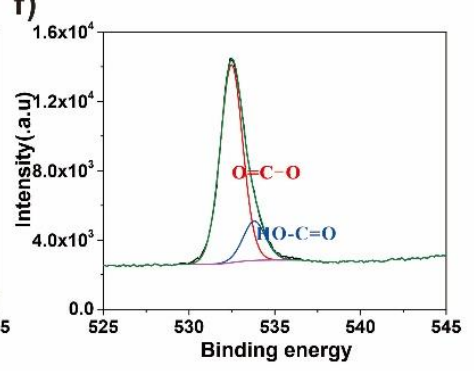

Figure S1. Full scale XPS spectra (a. d), high-resolution C 1s (b. e) and O 1s (c. f) spectrum of the carbon electrode. $(a, b, c)$ represent the untreated electrode, and $(d, e, f)$ represent the electrode after carboxyl activation.

a)

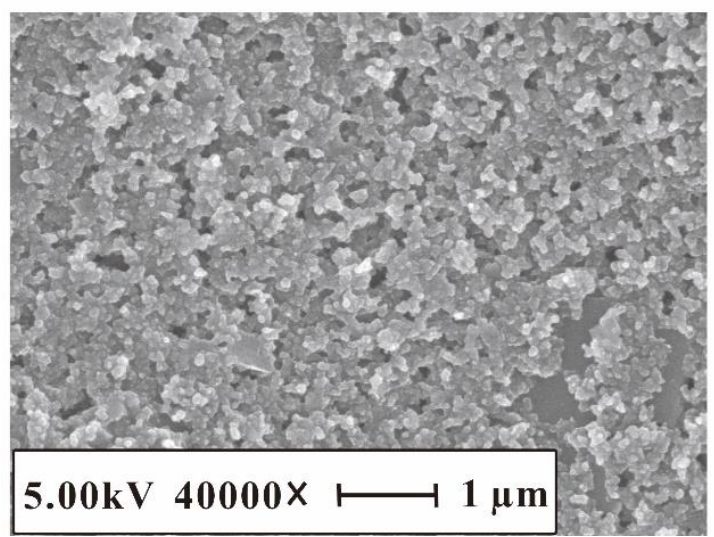

b)

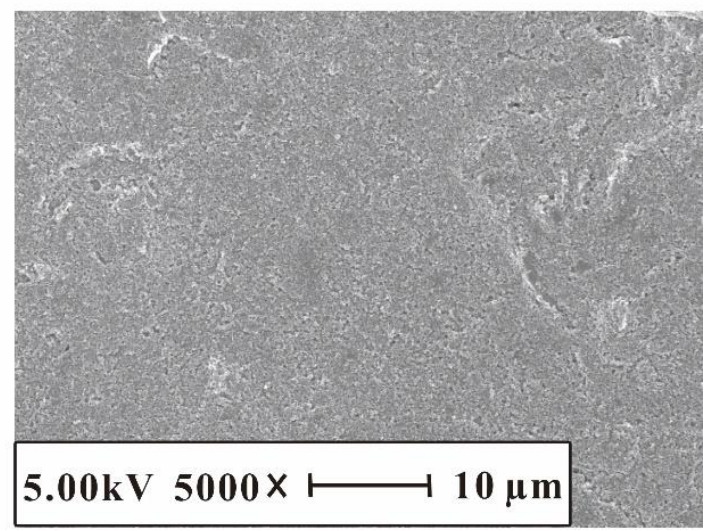

Figure S2. The scanning electron microscopic (SEM) image of the carbon surface. The magnifications of image a and $\mathrm{b}$ were 40000 and 5000 times, respectively. 
a)

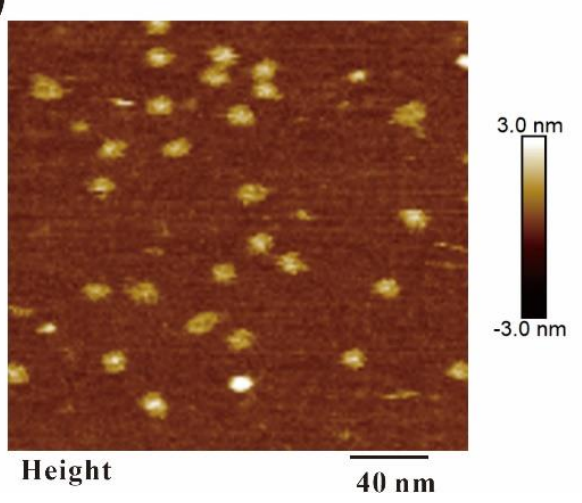

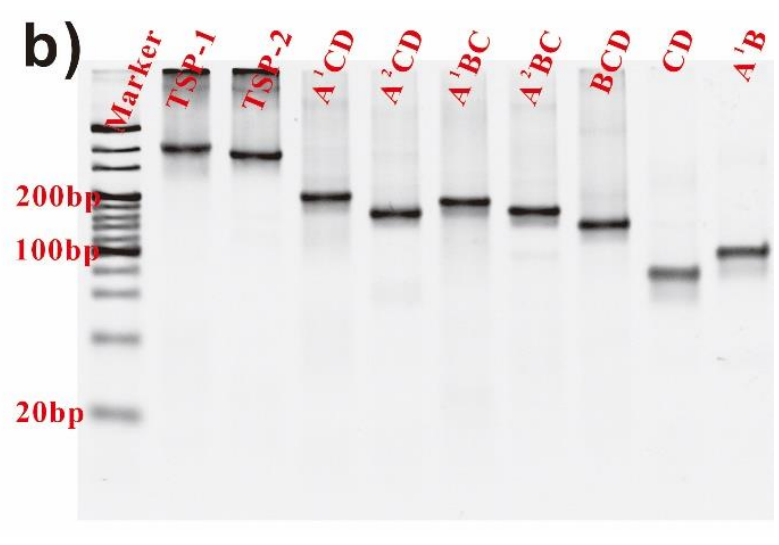

Figure S3. a) The atomic force microscopic (AFM) characterization of DNA tetrahedron on mica. b) polypropylene acyl amine gel electrophoresis (PAGE) analysis of DNA tetrahedron. TSP-1 and TSP-2 were respectively used for detection of ATP and miRNA. A1 and A2 were respectively the A strand of tetrahedron for ATP and miRNA.

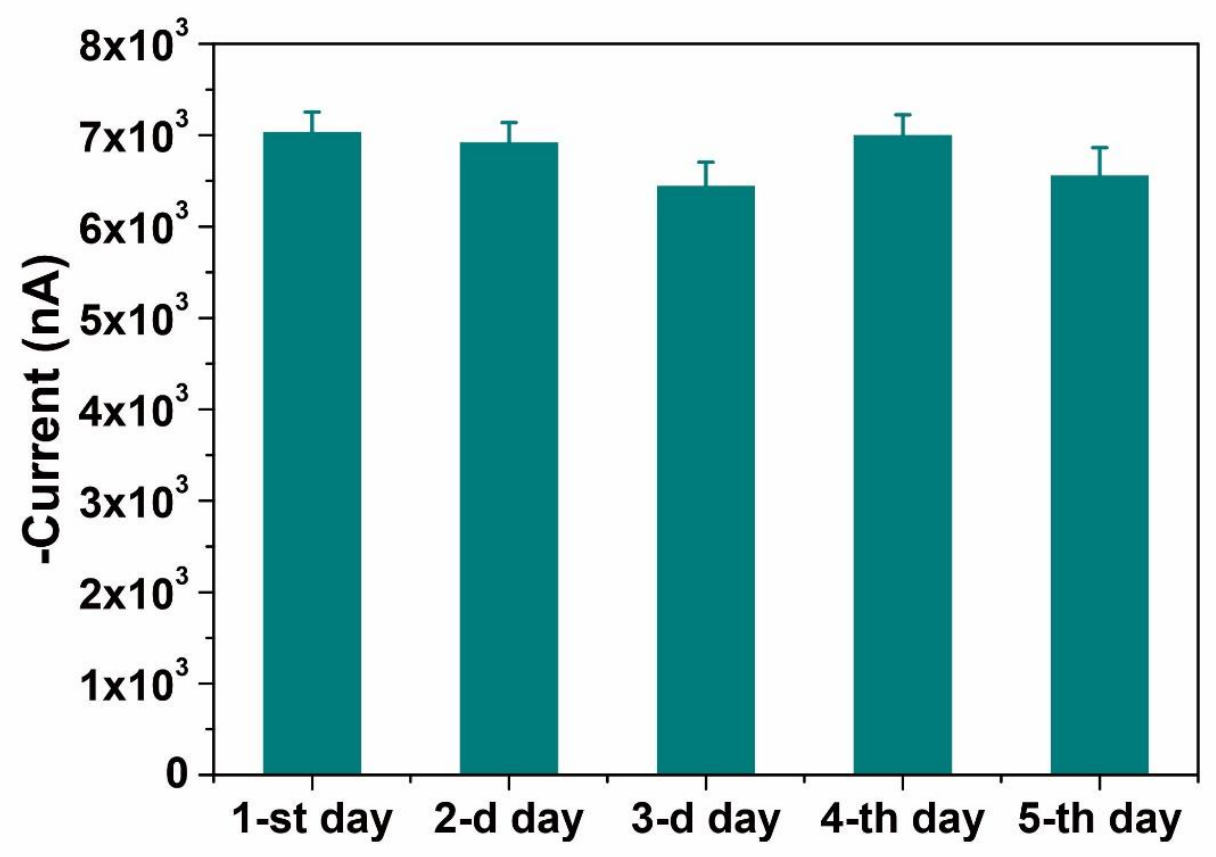

Figure S4. Repeatability of the DNA tetrahedron based biosensor. 
a)

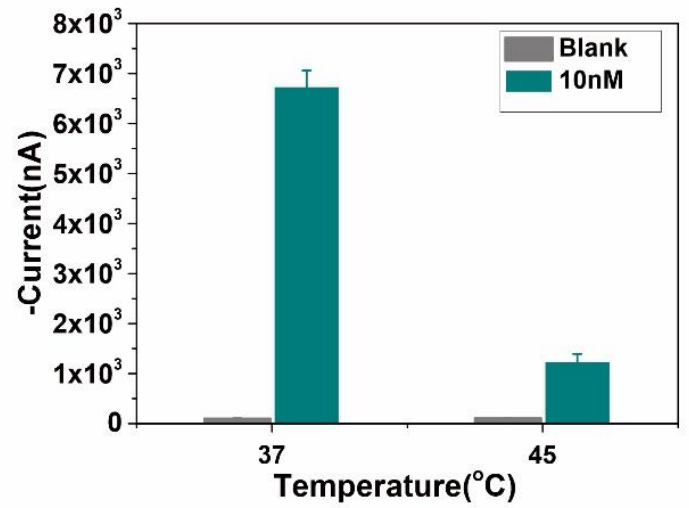

b)

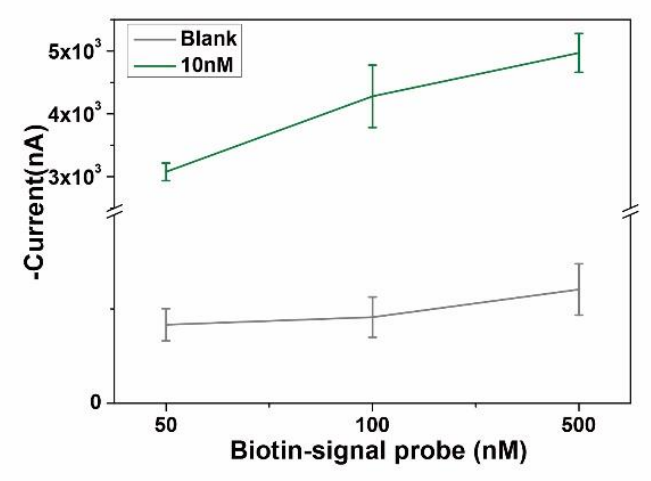

Figure S5. Optimization of reaction temperature (a) and the concentration of biotin-signal probe (b) for TSP modified carbon-based nano-bio electrochemical sensor (for 10nM of miRNA-141).

a)

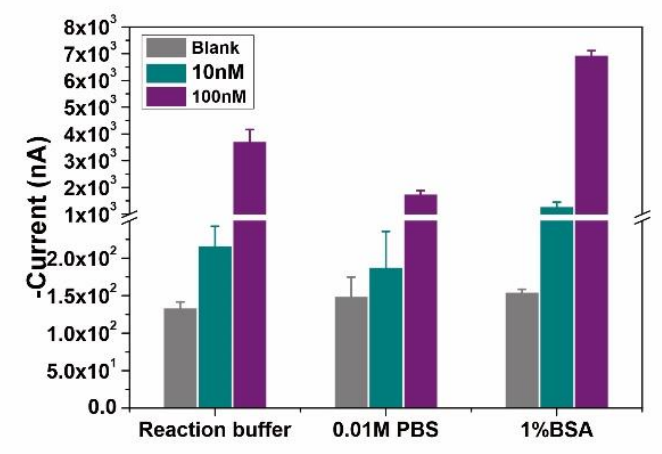

b)

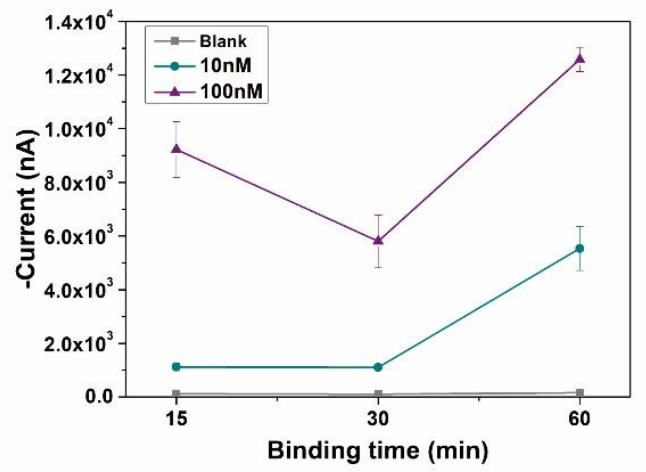

Figure S6. Optimization of reaction buffer (a) and the time (b) for TSP modified carbon-based nano-bio electrochemical sensor (for $10 \mathrm{nM}$ and $100 \mathrm{nM}$ of thrombin). Reaction buffer: ( $5 \mathrm{mM} \mathrm{KCl}, 100 \mathrm{mM} \mathrm{NaCl}, 50 \mathrm{mM}$ Tris ( $\mathrm{pH} 8.0$ ) ) 


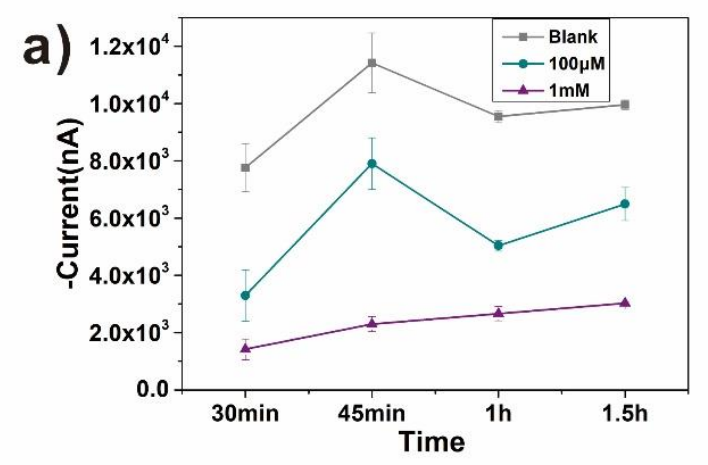

b)

c)
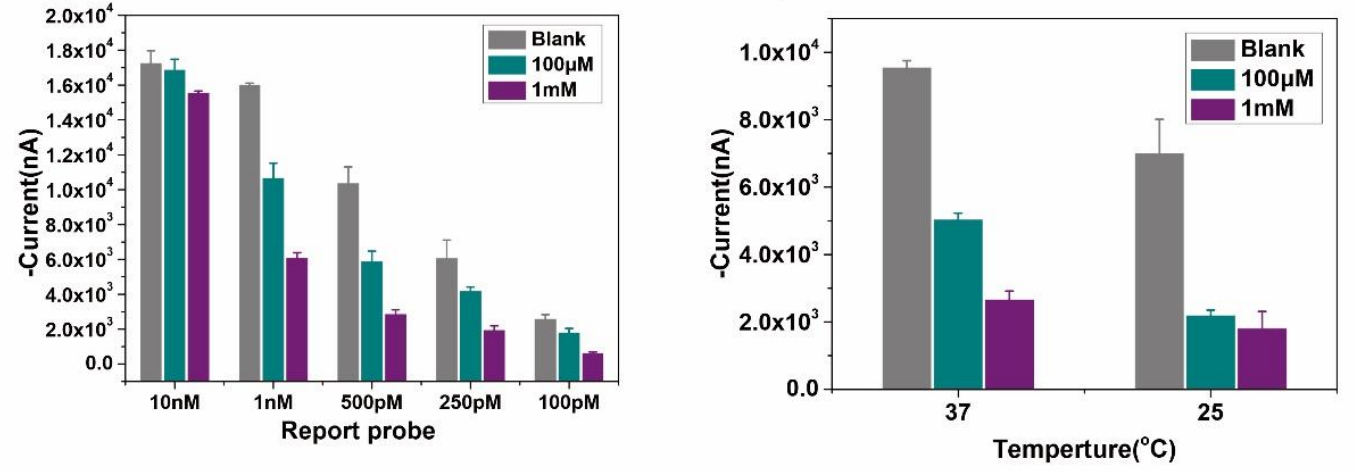

Figure S7. Optimization of reaction time (a), the concentration of report probe and the reaction temperature (c) for TSP modified carbon-based nano-bio electrochemical sensor (for $100 \mu \mathrm{M}$ and $1 \mathrm{mM}$ of ATP).

Table S1. DNA and MicroRNA Sequences Employed in This Work

\begin{tabular}{|l|l|}
\hline Oligonucleotide & sequence $\left(5^{\prime}-3^{\prime}\right)$ \\
\hline Tetra-B & NH2- TATCACCAGGCAGTTGACAGTGTAGCAAGCTGTAATAGATGCGAGGGTCCAATAC \\
\hline Tetra-C & NH2-TCAACTGCCTGGTGATAAAACGACACTACGTGGGAATCTACTATGGCGGCTCTTC \\
\hline Tetra-D & NH2-TTCAGACTTAGGAATGTGCTTCCCACGTAGTGTCGTTTGTATTGGACCCTCGCAT \\
\hline $\begin{array}{l}\text { Tetra-A(miRNA- } \\
141)\end{array}$ & $\begin{array}{l}\text { CATCTTGCCTAAAAAAAAAAACATTCCTAAGTCTGAAACATTACAGCTTGCTACACGAGAAGAG } \\
\text { CGCCATAGTA }\end{array}$ \\
\hline miRNA-141 & UAACACUGUCUGGUAAAGAUGG \\
\hline Gap-141 & AGGCAAGATGCCATCTTTACCAGACAGTGTTATGCGACCT \\
\hline Gap-21 & AGGCAAGATGTCAACATCAGTCTGATAAGCTATGCGACCT \\
\hline Gap-375 & AGGCAAGATGTCACGCGAGCCGAACGAACAAATGCGACCT \\
\hline SSP(miRNA-141) & CATCTTGCCTAAAAAAAAAA-NH2 \\
\hline Biotin(miRNA) & Biotin-AGGTCGCA \\
\hline Tetra-A(thrombin) & GGTTGGTGTGGTTGGAAAAAAACATTCCTAAGTCTGAAACATTACAGCTTGCTACACGAGAAGA \\
\hline
\end{tabular}




\begin{tabular}{|l|l|}
\hline & CCGCCATAGTA \\
\hline Biotin-Thrombin & Biotin-AGTCCGTGGTAGGGCAGGTTGGGGTGACT \\
\hline SSP (Thrombin) & GGTTGGTGTGGTTGGAAAAAA-NH2 \\
\hline Tetra-A (ATP) & $\begin{array}{l}\text { ACATTCCTAAGTCTGAAACATTACAGCTTGCTACACGAGAAGAGCCGCCATAGTATTTTTTAC } \\
\text { CTTCCTCCGCAATACTCCCCCAGGT }\end{array}$ \\
\hline Antisense (ATP) & Biotin-ACCTGGGGGAGTATTGCGGAGGAAGGT \\
\hline SSP(ATP) & NH2-TTTTTTACCTTCCTCCGCAATACTCCCCCAGGT \\
\hline Tetra-A-DNA & $\begin{array}{l}\text { GTATCCAGTGGCTCATTTTTTTTTTACATTCCTAAGTCTGAAACATTACAGCTTGCTACA } \\
\text { CGAGAAGAGCCGCCATAGTA }\end{array}$ \\
\hline ssDNA1 & GTATCCAGTGGCTCATTTTTTTTTT-NH2 \\
\hline Biotin-cDNA & TGAGCCACTGGATAC-Biotin \\
\hline Non-specific DNA & ACCTTCCTCCGCAATACTCCCCCAGGT-biotin \\
\hline
\end{tabular}

Table S2. Comparison of the properties of the conventional electrode with SPCE

\begin{tabular}{llll}
\hline & Properties & Conventional electrodes & SPCE array \\
\hline 1 & Price & Expensive & Cheap \\
2 & Disposability & No & Yes \\
3 & Combination with microfluidics & No & Yes \\
4 & High throughput & No & Yes \\
5 & Functionalization & Tough & Facile \\
6 & Biocompatibility & Yes/No & Yes \\
7 & Design & Difficult & Easy \\
\hline
\end{tabular}

